\title{
MONOTONIC NORMS IN ORDERED BANACH SPACES
}

\author{
K. F. NG AND C. K. LAW
}

(Received 7 October 1986)

Communicated by R. Vyborny

\begin{abstract}
Let $B$ be an ordered Banach space with ordered Banach dual space. Let $N$ denote the canonical half-norm. We give an alternative proof of the following theorem of Robinson and Yamamuro: the norm on $B$ is $\alpha$-monotone $(\alpha \geq 1)$ if and only if for each $f$ in $B^{*}$ there exists $g \in B^{*}$ with $g \geq 0, f$ and $\|g\| \leq \alpha N(f)$. We also establish a dual result characterizing $\alpha$-monotonicity of $B^{*}$.
\end{abstract}

1980 Mathematics subject classification (Amer. Math. Soc.): 46 A 40, 46 B 10.

Let $\left(B, B_{+},\|\cdot\|\right)$ be an ordered Banach space with closed cone (thus $a \leq b$ in $B$ means $\left.b-a \in B_{+}\right)$. Let $B^{*}$ denote the Banach dual space, ordered by the dual cone $B_{+}^{*}=\left\{f \in B^{*}: f(a) \geq 0\right.$ for all $\left.a \geq 0\right\}$. Let $N$ denote the canonical half-norms in $B$ or $B^{*}$. Thus

$$
\begin{aligned}
N(a) & :=\inf \{\|b\|: a \leq b \text { in } B\} \quad(a \in B) \\
& =\sup \left\{f(a): f \in B_{+}^{*},\|f\| \leq 1\right\}
\end{aligned}
$$

and

$$
\begin{aligned}
N(f) & :=\inf \left\{\|g\|: f \leq g \text { in } B^{*}\right\} \quad\left(f \in B^{*}\right) \\
& =\sup \left\{f(a): a \in B_{+},\|a\| \leq 1\right\} .
\end{aligned}
$$

The definition of $N$ is given by W. Arendt, P. R. Chernoff and T. Kato in [1], and the characterizations of $N$ are due to D. W. Robinson and S. Yamamuro [6, Theorems 2.1 and 3.5]. Theorem 1 below is essentially known:

(C) 1988 Australian Mathematical Society 0263-6115/88 \$A2.00 + 0.00 
(a) It was proved by Robinson and Yamamuro [6, Theorem 3.8] in the special case when $\alpha=1$.

(b) The equivalence (i) $\leftrightarrow$ (iv) was proved also in [2, Proposition 5]. Our proof given below seems to be more direct.

THEOREM 1. For $\alpha \geq 1$ the following conditions are equivalent:

(i) The norm is $\alpha$-monotone on $B$, that is, $0 \leq a \leq b$ in $B \rightarrow\|a\| \leq \alpha\|b\|$.

(ii) $\|a\| \leq \alpha N(a)+(\alpha+1) N(-a)$ for all $a$ in $B$.

(iii) For each $f$ in $B^{*}$ there exists $g \in B^{*}$ such that $0, f \leq g$ and $\|g\| \leq \alpha N(f)$.

(iv) For each $f$ in $B^{*}$ there exists $g \in B^{*}$ such that $f, 0 \leq g$ and $\|g\| \leq \alpha\|f\|$.

The verification for (i) $\rightarrow$ (ii) is as in (1) $\rightarrow$ (2) of Theorem 3.8 in [6]. Conversely, suppose (ii) holds and $0 \leq a \leq b$. Then $N(a) \leq\|b\|$ and $N(-a)=0$ by definition of $N$, so

$$
\|a\| \leq \alpha N(a)+(\alpha+1) N(-a) \leq \alpha N(a)+0 \leq \alpha\|b\| .
$$

Therefore (i) $\leftrightarrow$ (ii). That (iii) $\rightarrow$ (iv) is trivial. Assuming (iv), let $f \in B^{*}$ with $\|f\| \leq 1$ and take $g$ as in (iv). Then, whenever $0 \leq a \leq b$ in $B$, one has

$$
f(a) \leq g(a) \leq g(b) \leq \alpha\|f\|\|b\| \leq \alpha\|b\|
$$

and it follows from the Hahn-Banach theorem that $\|a\| \leq \alpha\|b\|$. Thus (iv) $\rightarrow$ (i), and it remains to prove (i) $\rightarrow$ (iii). We do this as in [4, Theorem 9.6]. Let $f \in B^{*}$. We define

$$
\begin{array}{ll}
q(a)=\sup \{f(b): 0 \leq b \leq a\} & \left(a \in B_{+}\right), \\
p(a)=\alpha N(f)\|a\| & (a \in B) .
\end{array}
$$

Then $q$ is superlinear, $p$ is sublinear and $q \leq p$ on $B_{+}$. By Bonsall's generalization of the Hahn-Banach theorem (see [4, Theorem 1.15]), there exists a linear functional $g$ on $B$ such that $q \leq g$ on $B_{+}$and $g \leq p$ on $B$. Then, for all $a \in B_{+}$,

$$
0, f(a) \leq q(a) \leq g(a)
$$

that is, $0, f \leq g$. Also, $g \leq p=\alpha N(f)\|\cdot\|$ on $B$ so $\|g\| \leq \alpha N(f)$, proving (iii).

REMARK. We have not used the completeness of $B$, that is, Theorem 1 is valid for ordered normed spaces. However, for the following dual result of Theorem 1, the completeness will be essential. (Again, the equivalence (i) $\leftrightarrow$ (iv) was known in the special case when $\alpha=1$, see [2, Proposition 6].)

THEOREM 2. For $\alpha \geq 1$ the following conditions are equivalent: (i) The norm is $\alpha$-monotone on $B^{*}$.

(ii) $\|f\| \leq \alpha N(f)+(\alpha+1) N(-f)$.

(iii) For each $a$ in $B$ and $\varepsilon>0$ there exists $b \in B$ with $0, a \leq b$ and $\|b\| \leq$ $\alpha N(a)+\varepsilon$. 
(iv) For each $a$ in $B$ and $\varepsilon>0$ there exists $b \in B$ with $0, a \leq b$ and $\|b\| \leq$ $\alpha\|a\|+\varepsilon$.

Proof. We need only prove (i) $\rightarrow$ (iii) as the other implications can be proved as in Theorem 1, or follow from Theorem 1 (applied to $B^{*}$ instead of $B$ ). Let $a \in B$. Define

$$
\begin{array}{ll}
q(f)=\sup \left\{g(a): 0 \leq g \leq f \text { in } B^{*}\right\} & \left(f \in B_{+}^{*}\right), \\
p(f)=\alpha N(a)\|f\| & \left(f \in B^{*}\right) .
\end{array}
$$

Then, as in the proof of [4, Theorem 9.7], we can verify that $p$ and $-q$ are lower semi-continuous sublinear functionals (respectively on $B^{*}$ and $B_{+}^{*}$ ) under the $w^{*}$-topology. By a result, dual to Bonsall's theorem (see [3, Theorem 3] or [4, Corollary 2.9]), for any $\varepsilon>0$, there exists $b$ in $B$ such that $q(f) \leq f(b)$ and $g(b) \leq p(g)+\varepsilon\|g\|$ for all $f \in B_{+}^{*}$, and $g \in B^{*}$. Then $g(b) \leq \alpha N(a)\|g\|+\varepsilon\|g\|$ and it follows from the Hahn-Banach theorem that $\|b\| \leq \alpha N(a)+\varepsilon$. Also, for all $f \in B_{+}^{*}, 0, f(a) \leq q(f) \leq f(b)$; since $B_{+}$is assumed to be closed it follows that $0, a \leq b$.

\section{References}

[1] W. Arendt, P. R. Chernoff and T. Kato, 'A generalization of dissipativity and positive semigroups', J. Operator Theory 8 (1982), 167-180.

[2] K. F. Ng, 'The duality of partially ordered Banach spaces', Proc. London Math. Soc. 19 (1969), 269-288.

[3] K. F. Ng, 'A note on partially ordered Banach spaces', J. London Math. Soc. (2) 1 (1969), $520-524$.

[4] Y. C. Wong and K. F. Ng, Partially ordered topological vector spaces, (Clarendon Press, Oxford, 1973).

[5] D. W. Robinson and S. Yamamuro, 'The Jordan decomposition and half-norms', Pacific J. Math. 110 (1984), 345-353.

[6] D. W. Robinson and S. Yamamuro, 'The canonical half-norm, dual half-norms and monotonic norms', Tôhoku Math. J. 35 (1983), 375-386.

Department of Mathematics

The Chinese University of Hong Kong

Hong Kong 